ARTICLE

\title{
Breaking a dative bond with mechanical forces
}

\author{
Pengcheng Chen ${ }^{1,9}$, Dingxin Fan ${ }^{2,9}$, Yunlong Zhang (10 ${ }^{3 凶}$, Annabella Selloni (i) ${ }^{4}$, Emily A. Carter (i) ${ }^{5,6}$, \\ Craig B. Arnold (10) ${ }^{1,5}$, David C. Dankworth (10 ${ }^{3}$, Steven P. Rucker (1) ${ }^{3}$, James R. Chelikowsky (D) ${ }^{2,7,8 凶} \&$ \\ Nan Yao (1) ${ }^{1 凶}$
}

Bond breaking and forming are essential components of chemical reactions. Recently, the structure and formation of covalent bonds in single molecules have been studied by noncontact atomic force microscopy (AFM). Here, we report the details of a single dative bond breaking process using non-contact AFM. The dative bond between carbon monoxide and ferrous phthalocyanine was ruptured via mechanical forces applied by atomic force microscope tips; the process was quantitatively measured and characterized both experimentally and via quantum-based simulations. Our results show that the bond can be ruptured either by applying an attractive force of $\sim 150 \mathrm{pN}$ or by a repulsive force of $220 \mathrm{pN}$ with a significant contribution of shear forces, accompanied by changes of the spin state of the system. Our combined experimental and computational studies provide a deeper understanding of the chemical bond breaking process.

\footnotetext{
${ }^{1}$ Princeton Institute for the Science and Technology of Materials, Princeton University, Princeton, NJ 08540-8211, USA. ${ }^{2}$ McKetta Department of Chemical Engineering, University of Texas at Austin, Austin, TX 78712-1589, USA. ${ }^{3}$ ExxonMobil Research and Engineering Company, Annandale, NJ 08801-3096, USA. ${ }^{4}$ Department of Chemistry, Princeton University, Princeton, NJ 08544-0001, USA. ${ }^{5}$ Department of Mechanical and Aerospace Engineering, Princeton University, Princeton, NJ 08544-5263, USA. ${ }^{6}$ Office of the Chancellor and Department of Chemical and Biomolecular Engineering, University of California, Los Angeles, Los Angeles, CA 90095-1405, USA. ${ }^{7}$ Department of Physics, University of Texas at Austin, Austin, TX 78712-1192, USA. ${ }^{8}$ Center for Computational Materials, Oden Institute for Computational Engineering and Sciences, University of Texas at Austin, Austin, TX 78712-1229, USA. ${ }^{9}$ These authors contributed equally: Pengcheng Chen, Dingxin Fan.凶email: nyao@princeton.edu; yunlong.zhang@exxonmobil.com; jrc@utexas.edu.
} 
T he ability to obtain images of organic molecules with atomic resolution was first demonstrated by Gross et al. in 2009 by using a carbon monoxide (CO) molecule attached to an Atomic Force Microscope (AFM) tip ${ }^{1}$ mounted on a qPlus sensor $^{2}$. This work inspired a wide range of applications, including directly characterizing molecular structures ${ }^{3-5}$, probing molecular properties ${ }^{6-10}$, creating new structures ${ }^{11,12}$, and even providing a tool for studying various types of chemical bonds, such as hydrogen bonds and halogen bonds ${ }^{13,14}$. These studies stimulated significant discussions on the contrast mechanism of AFM images and on the extent to which the image could represent a physical description of a chemical bond ${ }^{15,16}$. A similar technique was used to directly manipulate individual chemical bonds. Wagner et al. ${ }^{17}$ measured the binding energies (including nonspecific interactions) between an organic molecule, 3,4,9,10perylene-teracarboxylic-dianhydride, and a metal substrate, $\mathrm{Au}(111)$. Recently, Kawai et al. measured the $\mathrm{C}=\mathrm{O} \cdots \mathrm{H}-\mathrm{C}$ bonding interaction between a $\mathrm{CO}$ tip and the $\mathrm{C}-\mathrm{H}$ group of an aromatic hydrocarbon before the onset of Pauli repulsion ${ }^{18}$. Huber et al. studied the interactions between a $\mathrm{CO}$ tip with $\mathrm{Fe}$, $\mathrm{Cu}$, and $\mathrm{Si}$ adatoms and revealed the bond-forming process during the transition from physisorption to chemisorption ${ }^{19}$.

These studies using AFM tips to manipulate chemical bonds provided insights into the bond-forming process involved in many surface interactions. However, the controlled breaking of a chemical bond using mechanical forces, along with accurate measurements of these forces, is also important and its detailed process has not yet been fully understood. Understanding the process of rupturing a bond is essential for obtaining insights into the physical nature of a chemical bond and its role in many chemical and catalytic mechanisms. Dative bonds are commonly found in transition metal complexes and play vital roles in catalysis, organometallic chemistry, and biochemistry. Here, we focus on understanding the breaking of a single chemical bond between a CO molecule and a ferrous phthalocyanine $(\mathrm{FePc})$ complex using AFM together with real-space pseudopotential density functional theory (DFT) calculations ${ }^{20-22}$. Our results reveal detailed mechanisms of bond breaking by both repulsive and attractive forces. This work advances understanding of the origins of measured forces in dative bond breaking.

\section{Results}

STM/AFM topography and structure identification. We prepared a supported CO-FePc system by dosing $\mathrm{CO}$ molecules onto $\mathrm{FePc}$ adsorbed on a $\mathrm{Cu}(111)$ surface at $4.8 \mathrm{~K}$. The scanning tunneling microscope (STM) image in Fig. 1a shows two distinct features for the FePc molecule and the complex (CO-FePc), which are similar to previously reported STM images on other surfaces $^{23}$. AFM images were obtained using a CO-terminated tip, confirming their respective structures (Fig. 1b, c). The AFM image in Fig. $1 \mathrm{~b}$ of the $\mathrm{CO}-\mathrm{FePc}$ complex featured a protruding center due to the $\mathrm{CO}$ attached to $\mathrm{Fe}$. This characterization is confirmed by comparing with AFM images of FePc molecules on the surface Fig. 1c, and further verified by our simulated images (Fig. 1d, e).

Breaking a dative bond with AFM tips. The dative $\mathrm{CO}-\mathrm{FePc}$ bond is known to be formed via $\sigma$-donation from the CO $5 \sigma$ orbital and $\pi$-back donation from $\mathrm{Fe} d \pi^{24-27}$. We studied the rupture of this dative bond by applying mechanical forces using the AFM tip. The same CO-terminated tip employed for imaging was used first because it is known to be chemically inert ${ }^{28}$. By decreasing the tip height, the repulsive interactions increased, as indicated by the increased contrast in the images (Fig. 2b-d). At a tip height of about $+40 \mathrm{pm}$, the peripheral aromatic rings of $\mathrm{FePc}$ became visible, while the center of the image became distorted due to strong repulsions with the tip. Upon further reducing the tip height $(+30 \mathrm{pm})$, a sudden change of the image occurred during scanning, as indicated by a line created with a different contrast. Subsequent scans showed the repulsion had disappeared, indicating that the $\mathrm{CO}$ attached to FePc was dislodged due to the strong repulsion with the tip. The chemical structure of FePc revealed from subsequent scanning of the lower part of the molecule confirmed that a free FePc was left after $\mathrm{CO}$ removal and that the tip remained intact during the dissociation. Comparison of the contrast in the lower part to the upper part of the same AFM image Fig. 2d obtained at the same tip height reveals a downward shift of FePc by $\sim 30$ pm upon CO removal. This shift indicates a trans effect by the $\mathrm{Cu}$ substrate on the $\mathrm{FePc}$ complex ${ }^{24-27,29}$, whereby the binding or removal of one ligand
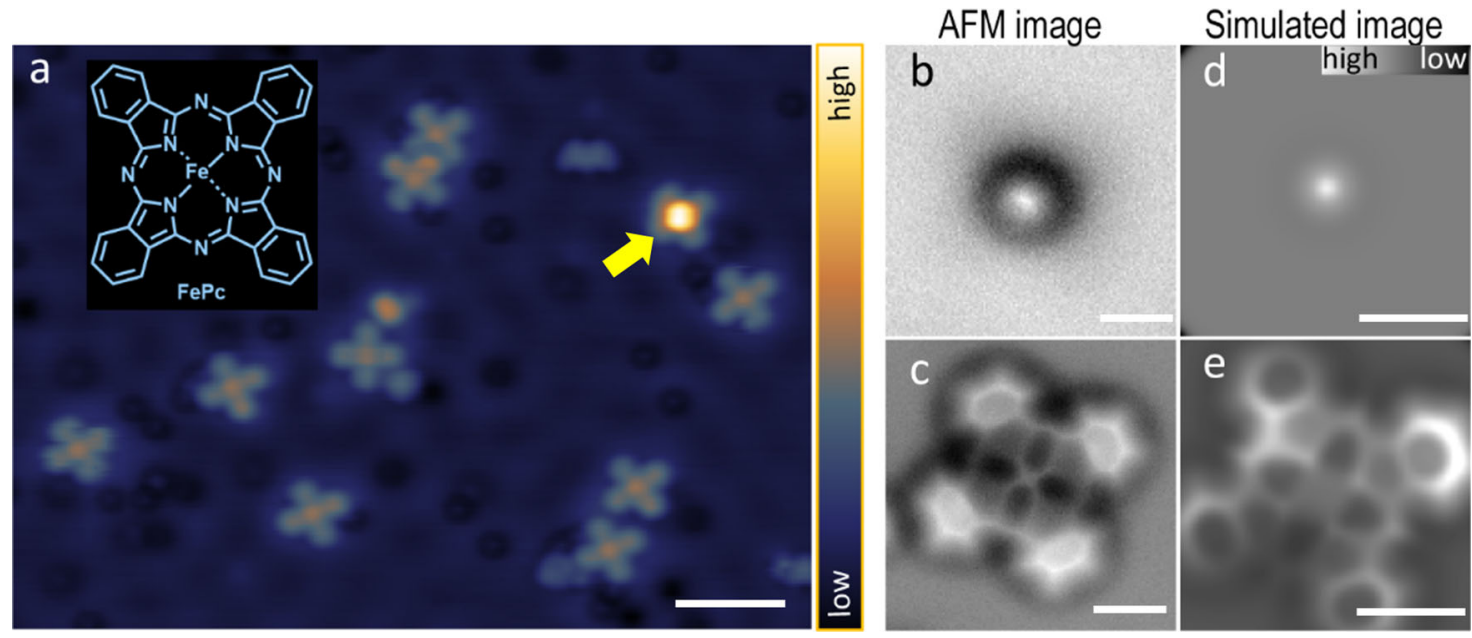

Fig. 1 STM and AFM images of FePc and the datively bonded CO-FePc complex. a STM image of the FePc molecule with (indicated by the arrow) and without adsorption of $\mathrm{CO}$, with the insert showing the chemical structure of FePc (set point: $V_{\text {sample }}=+100 \mathrm{mV}, \mathrm{I}=100 \mathrm{pA}, \mathrm{scale}$ bar: $3 \mathrm{~nm}$ ). b, $\mathbf{c}$ Experimental AFM images of FePc with and without adsorbed CO, obtained using a CO-terminated tip $(V=0 \mathrm{~V}, A=100 \mathrm{pm}$, scale bar: $0.5 \mathrm{~nm})$ at tip heights $z$ of $+160 \mathrm{pm}$ and $-10 \mathrm{pm}$, respectively. The tip height $z$ was set with respect to a reference height given by the STM set point (100 mV, $100 \mathrm{pA}$ ) above the bare $\mathrm{Cu}(111)$ substrate in the vicinity of the molecule. The minus sign of tip height $z$ indicates a decrease of tip height. $\mathbf{d}$, e Simulated AFM images corresponding to CO-FePc and FePc at tip heights of $554 \mathrm{pm}$ and $300 \mathrm{pm}$. The tip height in the simulation is defined as the distance between the front atom of the tip and the average height of the FePc complex (excluding the decorated CO) (scale bar: $0.5 \mathrm{~nm}$ ). 

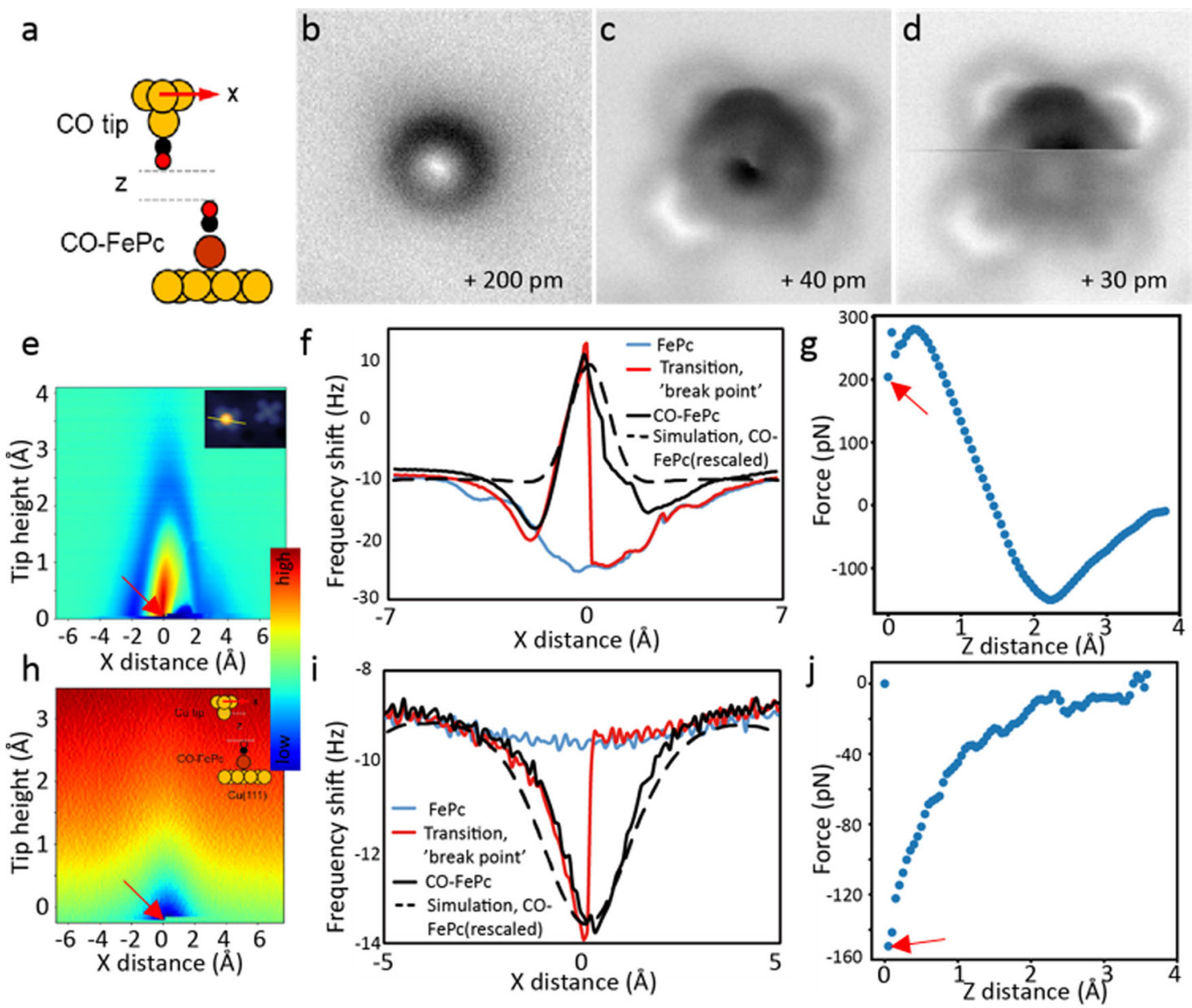

Fig. 2 Rupturing the dative CO-FePc bond using AFM tips. a Schematic of a CO-AFM tip interacting with CO-FePc (Cu: yellow; C: black; O: red; Fe: brown). b-d Non-contact AFM images obtained at different tip heights ( $\mathrm{z}$ ); the final dislodging of $\mathrm{CO}$ occurs at $z=+30 \mathrm{pm}$. e $3 \mathrm{D}$ force map of the frequency shift $(\Delta f)$ vs. AFM tip heights $(z)$ and horizontal position $(x)$, with a CO tip. Step size is $5 \mathrm{pm}$ in $z$, and the scan path in $x$ is across the center of the $\mathrm{Fe}$, as shown in the inset. The tip position at bond rupture is indicated by the breakpoint (arrow). f Frequency shift ( $\Delta f$ ) obtained in the horizontal ( $x$ ) direction before, during (indicated by the disjointed curve), and after the bond rupture. $\mathbf{g}$ The force curve deconvoluted from $\Delta f$ at the breakpoint in the vertical $(z)$ direction. $\mathbf{h} 3 \mathrm{D}$ force map of the frequency shift $(\Delta f)$ showing quantitative rupture of the dative bond, obtained using a Cu tip; the insert shows schematic of interaction between a Cu tip and CO-FePc. i Frequency shift ( $\Delta f$ ) obtained using a Cu tip scanned in the horizontal ( $x$ ) direction. $\mathbf{j}$ The deconvoluted force curve at the breakpoint in the vertical ( $z$ ) direction using a Cu tip. (Red arrows indicate the bond rupture point. Long-range background forces are subtracted in Figures $\mathbf{g}$ and $\mathbf{j}$ ).

respectively reduces or enhances the strength of the bond to the ligand on the opposite side ${ }^{30}$. This observation confirms the rupture of the dative bond between the $\mathrm{CO}$ and $\mathrm{FePc}$ induced by the increased interactions during tip scanning.

To elucidate $\mathrm{CO}-\mathrm{FePc}$ bond rupture, we performed detailed measurements of the interaction forces during the entire bond rupture process. Figure $2 \mathrm{e}$ shows a $3 \mathrm{D}$ force map representing the frequency shift $(\Delta f)$ obtained at different tip heights $(z)$ by scanning across the center of the CO-FePc complex (shown in the inset). The dislodging of the $\mathrm{CO}$ was indicated by a breakpoint $(x=0)$ with decreasing the tip height during scanning and by a discontinuity in the frequency shift $(\Delta f)$ curve (red curve in Fig. 2f). The interaction force and energy were calculated from the measured frequency shift with the method and formulas proposed by Sader ${ }^{31}$. The force curve along with the tip height $(\mathrm{z})$ at the breaking point $(x=0)$, in Fig. $2 \mathrm{~g}$, shows that the dative bond ruptured with a force of $220 \pm 30 \mathrm{pN}$, after passing a maximal force at $\sim 300 \mathrm{pN}$.

Dislodging experiments were also performed with a bare metal tip, which was terminated by a $\mathrm{Cu}$ atom under similar experimental conditions as for the CO-terminated tip. The $\mathrm{Cu}$ atom tip is known to be a chemically active tip ${ }^{32}$. When we used the $\mathrm{Cu}$ tip, only attractive interactions between the tip and COFePc were detected (Fig. 2h), until the rupture of the dative bond took place. At this point, the attractive force reached $150 \pm 30 \mathrm{pN}$ by reducing the tip height (Fig. $2 \mathrm{i}, \mathrm{j}$ ).

Real-space DFT calculations. The surprising observation that both an attractive force of $-150 \mathrm{pN}$ and a repulsive force of $+220 \mathrm{pN}$ are capable of breaking the same dative bond highlights the important role of probe tips, although this result is consistent with findings by Berwanger et al. that the CO-terminated tip can exert forces of up to $450 \mathrm{pN}$ without breaking off ${ }^{33,34}$. We employed real-space DFT calculations to address the role of the AFM tip and to shed light on the details of the bond-breaking process.

To understand interactions between the AFM tips and COFePc before the bond is broken, we computed frequency shifts of the probe tips at relatively large heights $(\mathrm{z} \sim 5 \AA)$ using optimized geometries $^{22}$ (Supplementary Figure 1). We modeled the $\mathrm{Cu}$ tip using a $\mathrm{Cu}_{2}$ cluster and the $\mathrm{CO}$ tip using a $\mathrm{Cu}-\mathrm{CO}$ cluster. We tested more complex tip conformations and found the effect in 

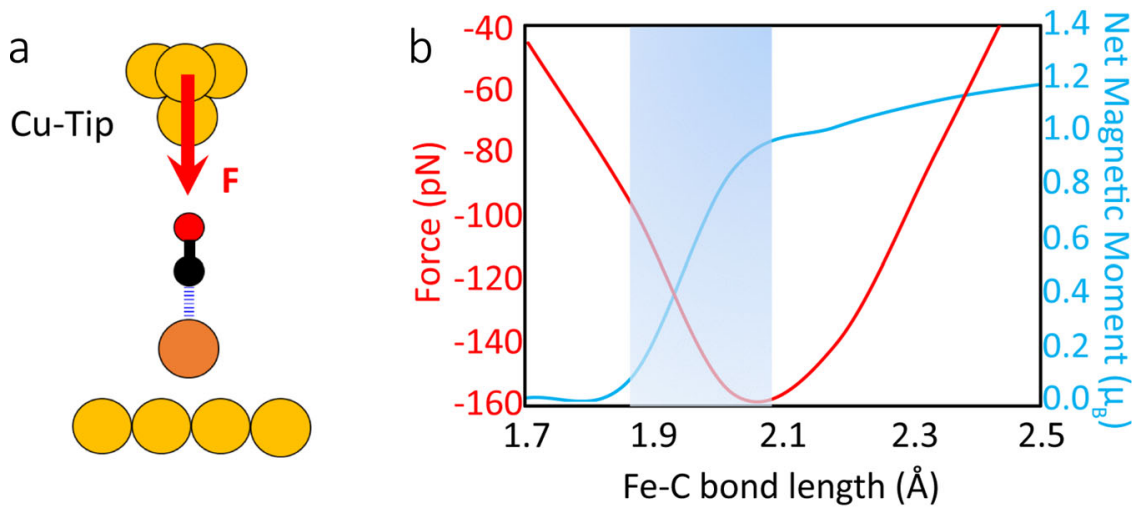

Fig. 3 Real-space pseudopotential DFT calculations for the breaking of the dative bond in CO-FePc with a Cu tip. a Schematic showing the interaction of the Cu tip with the CO-FePc complex on $\mathrm{Cu}$ (111) (Cu: yellow; C: black; O: red; Fe: brown). The red arrow indicates the attractive force acting on the tip apex. b The red curve shows the calculated attractive vertical force on the $\mathrm{Cu}$ apex while the blue curve shows the net magnetic moment as a function of the $\mathrm{Fe}-\mathrm{C}$ bond length. The shaded area indicates where the bond rupture process occurred.
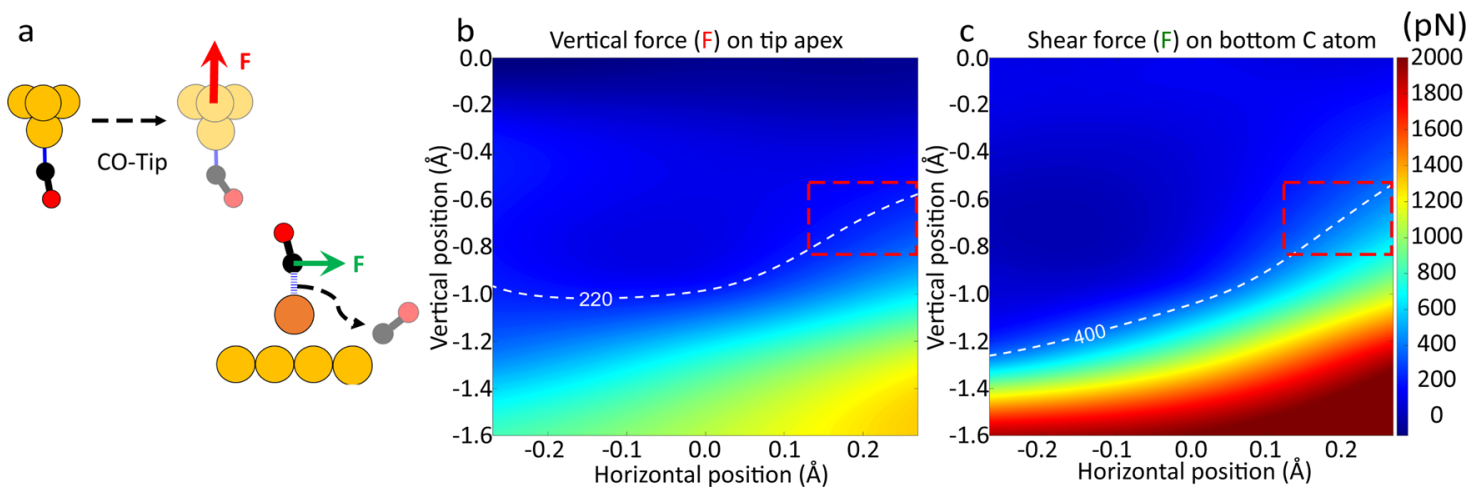

Fig. 4 Real-space pseudopotential DFT calculations for the breaking of the dative bond in CO-FePc with a CO tip. a Schematic shows the interactions between the $\mathrm{CO}$ tip and the $\mathrm{CO}-\mathrm{FePc}$ complex on $\mathrm{Cu}(111)$. The red and green arrows indicate the direction of the forces acting on the tip apex and on the bottom $\mathrm{C}$ atom, respectively. (Cu: yellow; C: black; O: red; Fe: brown) b, c are line cuts of 3D force map for the calculated compressive vertical force on the tip apex, and the lateral force acting on the bottom $\mathrm{C}$ (of the $\mathrm{CO}$ attached to $\mathrm{FePc}$ ). The $\mathrm{x}$ and $\mathrm{y}$ axes are the horizontal positions and heights of the tip with respect to an equilibrium position. The dashed curves in $\mathbf{b}$ and $\mathbf{c}$ correspond to the measured force, $220 \mathrm{pN}$, and where the shear force approaches $-400 \mathrm{pN}$. The red dashed boxes indicate the same region in $\mathbf{b}$ and $\mathbf{c}$ where the dative bond is most likely to be ruptured.

tip-sample interaction energy, whose gradient is proportional to the forces, is negligible (Supplementary Figure 2). The relative frequency shifts from calculations are in excellent agreement with the measured curves for both $\mathrm{CO}$ and $\mathrm{Cu}$ tips (Fig. 2f, i). To simulate the approach of an AFM tip toward the sample as conducted experimentally, we reduced the tip heights until optimal tip-sample distances are found (see Supplementary Methods, section 3 for details). We first examined the $\mathrm{Cu}$ tip (Fig. 3a), which is less complicated than the CO tip due to the multidimensional tilt of the two CO molecules for the CO tip (see Supplementary Figure 3, and Supplementary Data 1-6). We find the vertical force acting on the $\mathrm{Cu}$ tip base $\left(F_{\mathrm{z}}\right)$ is attractive, reaches a maximum of $-156 \mathrm{pN}$. This agrees with the experimentally measured force of $-150 \pm 30 \mathrm{pN}$ very well (Fig. 3b). In addition, a transition from low to high spin occurs when the Fe-C bond is stretched to $\sim 1.9-2.1 \AA$ (shaded area in Fig. 3b), indicating the rupture of the dative bond in $\mathrm{CO}-\mathrm{FePc}$ reported in a previous study ${ }^{30}$. When applying the same computational method to the $\mathrm{CO}$ tip, we find the dative bond in $\mathrm{CO}-\mathrm{FePc}$ is not broken by decreasing the tip height $z$ when a compressive vertical force $\left(F_{z}\right)$ is applied on top of the center of the $\mathrm{CO}-\mathrm{FePc}$ complex $(x=0)$. In our calculation, we displaced the CO tip horizontally $(x \neq 0)$ while decreasing the tip height, similar to the experimental scanning of AFM tip (Fig. 4a). Here, we performed additional structural relaxation calculations as we moved the CO tip away from the center to ensure our equilibrium structure represents a reasonable geometry (see Supplementary Methods, section 3.2 for details). Significantly, we find that a small increase in the vertical force $\left(F_{z}\right)$ acting on the tip apex (Fig. 4b) results in a rapid increase of the lateral (shear) force $\left(F_{\mathrm{x}}\right)$ acting on the $\mathrm{C}$ atom in the CO-FePc complex (Fig. 4c) at low tip heights. However, we believe the dative bond is ruptured before the shear force could reach a few $\mathrm{nN}$. To locate the approximate tip positions where the dative bond is ruptured, we also explored the space around the $\mathrm{CO}-\mathrm{FePc}$ complex with detailed calculations (dashed curves inside the red boxes). We deduce that the bond is ruptured when the shear force on $\mathrm{C}$ reaches about $400 \mathrm{pN}$ by fixing the compressive force $\left(F_{\mathrm{z}}\right)$ exerted on the tip apex at $220 \mathrm{pN}$ (the experimentally measured force). We find a shear force of $400 \mathrm{pN}$ is a reasonable value, given the fact that the dative bond in $\mathrm{CO}-\mathrm{FePc}$ is weakened by the $\mathrm{Cu}$ substrate due to the trans effect. We conclude that the dative bond is ruptured by a lateral force when a compressive force $(+220 \mathrm{pN})$ is applied by the CO-terminated tip.

The calculated spin-polarized local density of states projected onto the center Fe atom of the system shows that the removal of $\mathrm{CO}$ results in an increase (from 0.00 to $1.20 \mu_{\mathrm{B}}$ ) in the net magnetic moment (Fig. 5a, b). This change in spin state is 

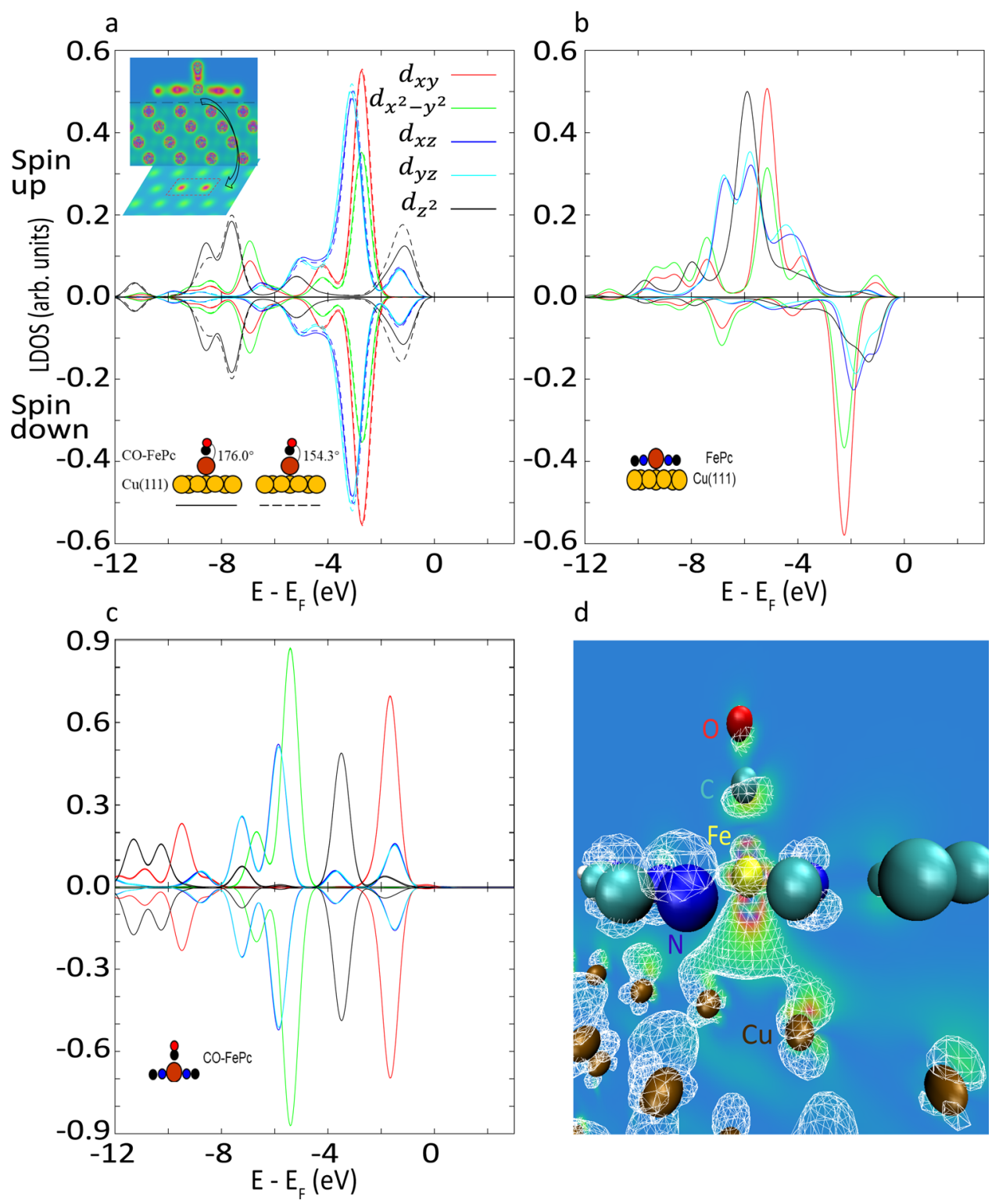

Fig. 5 The spin-polarized local density of states projected onto the center Fe atom. a $\mathrm{CO}-\mathrm{FePc}$ on $\mathrm{Cu}(111)$ with two $\mathrm{O}-\mathrm{C}-\mathrm{Fe}$ angles, $176^{\circ}$ (at equilibrium, solid curves) and $154^{\circ}$ (manually rotated, $\mathrm{Fe}-\mathrm{C}$ and $\mathrm{C}=\mathrm{O}$ bond lengths are fixed during rotation, dashed curves), net magnetic moment $=0.0 \mu_{\mathrm{B}}$. $\mathbf{b} \mathrm{FePc}$ on $\mathrm{Cu}(111)$, net magnetic moment $=1.2 \mu_{\mathrm{B}}$. c CO-FePc without a substrate, net magnetic moment $=0.0 \mu_{\mathrm{B}}$. In $\mathbf{a}$, the top inset shows a $2 \mathrm{D}$ vertical profile of the total electron density across the Fe atom. The black arrow indicates a 2D horizontal profile in between the complex and the Cu surface where the red dashed rectangle encloses the two bridge $\mathrm{Cu}$ atoms underneath the center Fe. $\mathbf{d}$ The HOMO of the system (around the center Fe atom). A wireframe view of the orbitals is overlapped with a 2D vertical profile of the electron density. Electron density figures are presented in Red-Green-Blue scale (red/blue: higher/lower electron density). Isosurface value $=10^{-5} \mathrm{e} / \mathrm{bohr}{ }^{3}$.

consistent with Fig. $3 \mathrm{~b}$ and a previous DFT study of the CO-FePc complex on the $\mathrm{Au}(111)$ surface $^{30}$. The total electron density and the highest occupied molecular orbital (HOMO) of the CO-FePc system demonstrate that the $\mathrm{Fe}$ center interacts with the two bridge $\mathrm{Cu}$ atoms underneath via the $d_{z}^{2}$ orbital (Fig. $5 \mathrm{a}, \mathrm{d}$ ). Figure $5 \mathrm{a}, \mathrm{c}$ shows the presence of the $\mathrm{Cu}(111)$ substrate weakens the $\mathrm{Fe}-\mathrm{C}$ dative bond by shifting the $d_{z}{ }^{2}$ orbital toward the HOMO, confirming the trans effect by the $\mathrm{Cu}$ substrate. To better understand the mechanisms of the bond rupture process, we manually changed the $\mathrm{O}-\mathrm{C}-\mathrm{Fe}$ bond angle by rotating the $\mathrm{CO}$ from its equilibrium position (from $176^{\circ}$ to $154^{\circ}$ ) and computed the orbital contribution around the center Fe atom (Fig. 5a). Our choice of the rotated symmetry is not unique, Supplementary Figure 5 shows the results of another bond angle. We find that breaking the almost linear symmetry $\left(176^{\circ}\right)$ of the $\mathrm{O}-\mathrm{C}-\mathrm{Fe}$ bond shifts the $\sigma$-component $\left(d_{z}^{2}\right)$ from lower energy states to higher energy states, while changes in the $\pi$-components are negligible.

\section{Discussion}

Our measurements show that the dative bond in the CO-FePc complex can be ruptured by either a $\mathrm{Cu}$ tip, or by a $\mathrm{CO}$ tip. These two tips bring out different mechanisms in bond breaking, i.e., an attractive force of $-150 \mathrm{pN}$ by a $\mathrm{Cu}$ tip, and a repulsive force of +220 pN by a CO tip. Our real-space DFT calculations accurately predict both the magnitudes and directions of the applied forces exerted on the tip apex $\left(F_{z}\right)$. These calculations also reveal that the dative bond is ruptured directly by a vertical force in the case of the $\mathrm{Cu}$ tip while the rupture is more likely caused by a lateral shear force when a repulsive force is applied by a CO tip. We also examined the possible influence of different tip geometries, 
finding that more complex tips do not affect significantly the tipsample interaction energy. As for the equilibrium conformation of the CO tip, our calculation is in agreement with previous studies 35,36

Our calculations confirm that the dative $\mathrm{CO}-\mathrm{FePc}$ bond is weakened by the presence of the $\mathrm{Cu}(111)$ substrate due to the trans effect ${ }^{29,30}$. However, the CO-FePc bond remains a chemical bond, instead of a physisorption ${ }^{19}$, based on the computed $\mathrm{Fe}-\mathrm{C}$ bond length $(\sim 1.7 \AA)$. Furthermore, upon the dislodging of the CO by the AFM tip, a $\sim 30$ pm downward shift of FePc observed in the experiment revealed that the $\mathrm{FePc}$ molecule was lifted $\sim 30 \mathrm{pm}$ upward from $\mathrm{Cu}(111)$ surface by attaching a single $\mathrm{CO}$ molecule to Fe. This observation is consistent with our calculated shortening of the distance between the Fe atom and the surface of the $\mathrm{Cu}$ substrate from 273 to $248 \mathrm{pm}$, further confirming the rupture of the chemical bond.

When a CO tip is used, the dative bond ruptures at $220 \mathrm{pN}$, after passing a maximal force of about $300 \mathrm{pN}$, as shown in Fig. $2 \mathrm{~g}$. This trend is consistent with a sequential cleavage of the $\sigma$-donation and $\pi$-back donation of the dative bond as Fig. $5 \mathrm{a}$ shows. The $\sigma$-bond has a higher symmetry than the $\pi$-bond and hence a higher force is required to tilt and weaken the $\sigma$-bond before the dative bond is completely ruptured by the lateral force.

Metal-Pc molecules are widely used model catalysts system for electrochemical reduction of $\mathrm{CO}_{2}{ }^{37,38}$. Our study is important for understanding how the activities of Metal-Pc molecules can be manipulated and controlled with single atomic level engineering, and for the design of new FePc-based catalysts. In addition, our results clarify the different mechanisms in bond breaking induced by inert and active tips. This detailed information on the rupture of the bond between $\mathrm{CO}$ and $\mathrm{FePc}$ will allow us to better understand other dative bonds, such as CO-heme interactions in biochemistry ${ }^{24,39}$, as well as chemical reactions of materials under mechanical stress ${ }^{40,41}$.

\section{Methods}

Experimental parameters. Our experiments were performed with a $\mathrm{CreaTec}^{\mathrm{TM}}$ STM/AFM system under ultrahigh vacuum conditions of $\sim 10^{-10} \mathrm{mbar}$ and a temperature of approximately $5 \mathrm{~K}$. The qPlus sensor has a resonance frequency of $30 \mathrm{kHz}$ with a spring constant $k=1800 \mathrm{~N} / \mathrm{m}$. In our measurements, the quality factor of the sensor is about 20,000. To minimize crosstalk between the qPlus signal and the STM channel, no voltage was applied on the tip during the force measurement process. The oscillation amplitude was set to be $100 \mathrm{pm}$.

Chemicals and sample preparation. The iron(II) phthalocyanine (FePc, dye content $\sim 90 \%$, Sigma-Aldrich) molecules were evaporated from a silicon chip via direct heating, and the vapor was subsequently deposited on a $\mathrm{Cu}(111)$ substrate held at $5 \mathrm{~K}$. The AFM Cu tip apex was functionalized by controlled pickup of a CO molecule from the substrate ${ }^{1}$. All the experiments were conducted using a pure $\mathrm{Cu}$ tip or a CO-functionalized tip.

DFT modelling and computations. We computed ground state energies using a real-space pseudopotential DFT code, PARSEC $^{42}$. We employed the local density approximation (LDA) by Perdew-Wang (PW92) ${ }^{43}$ for the exchange-correlation functional together with Troullier-Martins norm-conserving pseudopotentials ${ }^{44}$. We also tested another exchange-correlation functional by Ceperley-Alder ${ }^{45}$, the differences were negligible. In addition, a previous study showed that LDA and the generalized gradient approximation (GGA) gave similar results for properties of $\mathrm{FePc}$ and $\mathrm{CO}-\mathrm{FePc}$ on $\mathrm{Au}(111)^{30}$. We employed boundary conditions that require the electron wave functions to vanish outside a spherical or a slab domain, of which the boundary is at least $\sim 300 \mathrm{pm}$ from the outermost atom. We set the distance between neighbor points in the real-space grid to be $16 \mathrm{pm}$. The density-weighted self-consistent residual error was less than $10^{-4} \mathrm{Ry}$. We employed a finite difference method to approximate the relative frequency shift profiles based on the computed ground state energies across the middle line of the FePc complex, as indicated in Fig. 2f, i at relatively large tip heights. We performed further structural relaxations when the tips were close to the specimen, as the assumption that the movement of the tip had negligible influence on the electronic structure of the specimen may not be valid. We then applied the Hellmann-Feynman theorem to the total ground state energies to compute the net forces acting on each atom. We employed the frozen density embedding theory and a finite difference method for
AFM image simulations ${ }^{22}$ (see Supplementary Information-Image Simulations section and Supplementary Fig. 4 for details).

\section{Data availability}

The data supporting our results are available within this article and the Supplementary Information. The Supplementary Information contains a more detailed description of force calculation, structural relaxation, AFM image simulations and tip conformation tests. In addition, we provide the relaxed atomic coordinates of the systems in Supplementary Figure 3 and in Supplementary Data 1-6.

Received: 4 March 2021; Accepted: 2 September 2021; Published online: 24 September 2021

\section{References}

1. Gross, L., Mohn, F., Moll, N., Liljeroth, P. \& Meyer, G. The chemical structure of a molecule resolved by atomic force microscopy. Science 325, 1110 LP-1111114 (2009).

2. Giessibl, F. J. High-speed force sensor for force microscopy and profilometry utilizing a quartz tuning fork. Appl. Phys. Lett. 73, 3956-3958 (1998).

3. Gross, L. et al. Organic structure determination using atomic-resolution scanning probe microscopy. Nat. Chem. 2, 821-825 (2010).

4. Schuler, B. et al. Heavy oil based mixtures of different origins and treatments studied by atomic force microscopy. Energy Fuels 31, 6856-6861 (2017).

5. Hanssen, K. Ø. et al. A combined atomic force microscopy and computational approach for the structural elucidation of breitfussin A and B: highly modified halogenated dipeptides from Thuiaria breitfussi. Angew. Chem. Int. Ed. 51, 12238-12241 (2012)

6. Gross, L. et al. Bond-order discrimination by atomic force microscopy. Science 337, 1326-1329 (2012).

7. Fatayer, S. et al. Molecular structure elucidation with charge-state control. Science 365, 142-145 (2019).

8. Gross, L. et al. Measuring the charge state of an adatom with noncontact atomic force microscopy. Science 324, 1428-1431 (2009).

9. Mohn, F., Gross, L., Moll, N. \& Meyer, G. Imaging the charge distribution within a single molecule. Nat. Nanotechnol. 7, 227-231 (2012).

10. de Oteyza, D. G. et al. Noncovalent dimerization after enediyne cyclization on Au(111). J. Am. Chem. Soc. 138, 10963-10967 (2016).

11. Pavliček, N. et al. Synthesis and characterization of triangulene. Nat. Nanotechnol. 12, 308-311 (2017).

12. Kaiser, K. et al. An sp-hybridized molecular carbon allotrope, cyclo[18] carbon. Science 365, 1299-1301 (2019).

13. Zhang, J. et al. Real-Space identification of intermolecular bonding with atomic force microscopy. Science 342, 611-614 (2013).

14. Han, Z. et al. Imaging the halogen bond in self-assembled halogenbenzenes on silver. Science 358, 206-210 (2017).

15. Ellner, M., Pou, P. \& Pérez, R. Molecular identification, bond order discrimination, and apparent intermolecular features in atomic force microscopy studied with a charge density based method. ACS Nano 13 786-795 (2019).

16. Hämäläinen, S. K. et al. Intermolecular contrast in atomic force microscopy images without intermolecular bonds. Phys. Rev. Lett. 113, 186102 (2014).

17. Wagner, C. et al. Measurement of the binding energies of the organic-metal perylene-teracarboxylic-dianhydride/Au(111) bonds by molecular manipulation using an atomic force microscope. Phys. Rev. Lett. 109, 076102 (2012).

18. Kawai, $\mathrm{S}$. et al. Direct quantitative measurement of the $\mathrm{C}=\mathrm{O} \cdots \mathrm{H}-\mathrm{C}$ bond by atomic force microscopy. Sci. Adv. 3, e1603258 (2017)

19. Huber, F. et al. Chemical bond formation showing a transition from physisorption to chemisorption. Science 366, 235-238 (2019).

20. Sakai, Y., Lee, A. J. \& Chelikowsky, J. R. First-principles atomic force microscopy image simulations with density embedding theory. Nano Lett. 16, 3242-3246 (2016)

21. Chelikowsky, J. R., Fan, D., Lee, A. J. \& Sakai, Y. Simulating noncontact atomic force microscopy images. Phys. Rev. Mater. 3, 110302 (2019)

22. Fan, D., Sakai, Y. \& Chelikowsky, J. R. Chemical and steric effects in simulating noncontact atomic force microscopy images of organic molecules on a $\mathrm{Cu}$ (111) substrate. Phys. Rev. Mater. 4, 53802 (2020).

23. Tsukahara, N., Minamitani, E., Kim, Y., Kawai, M. \& Takagi, N. Controlling orbital-selective Kondo effects in a single molecule through coordination chemistry. J. Chem. Phys. 141, 54702 (2014).

24. Stynes, D. V. \& James, B. R. Kinetics and equilibriums for carbon monoxide binding to ferrous phthalocyanine complexes. J. Am. Chem. Soc. 96, 2733-2738 (1974) 
25. Calderazzo, F. et al. Synthesis and Moessbauer spectroscopic studies of carbonyl derivatives of (phthalocyaninato)iron(II). Inorg. Chem. 21, 2302-2306 (1982).

26. Li, J., Noll, B. C., Schulz, C. E. \& Scheidt, W. R. Comparison of cyanide and carbon monoxide as ligands in iron(II) porphyrinates. Angew. Chem. Int. Ed. 48, 5010-5013 (2009).

27. Collman, J. P., Brauman, J. I. \& Doxsee, K. M. Carbon monoxide binding to iron porphyrins. Proc. Natl Acad. Sci. 76, 6035-6039 (1979).

28. Sang, $H$. et al. Identifying tips for intramolecular NC-AFM imaging via in situ fingerprinting. Sci. Rep. 4, 6678 (2014).

29. Hieringer, W. et al. The surface trans effect: influence of axial ligands on the surface chemical bonds of adsorbed metalloporphyrins. J. Am. Chem. Soc. 133, 6206-6222 (2011).

30. Isvoranu, C. et al. Comparison of the carbonyl and nitrosyl complexes formed by adsorption of $\mathrm{CO}$ and $\mathrm{NO}$ on monolayers of iron phthalocyanine on Au(111). J. Phys. Chem. C. 115, 24718-24727 (2011).

31. Sader, J. E. \& Jarvis, S. P. Accurate formulas for interaction force and energy in frequency modulation force spectroscopy. Appl. Phys. Lett. 84, 1801 (2004).

32. Mönig, H. Copper-oxide tip functionalization for submolecular atomic force microscopy. Chem. Commun. 54, 9874 (2018).

33. Berwanger, J. et al. Atomically resolved chemical reactivity of small Fe clusters. Phys. Rev. Lett. 124, 096001 (2020).

34. Berwanger, J., Huber, F., Stilp, F. \& Giessibl, F. J. Lateral manipulation of single iron adatoms by means of combined atomic force and scanning tunneling microscopy using CO-terminated tips. Phys. Rev. B 98, 195409 (2018).

35. Weymouth, A. J., Hofmann, T. \& Giessibl, F. J. Quantifying molecular stiffness and interaction with lateral force microscopy. Science 343, 1120-1122 (2014).

36. Salmeron, M. CO meets CO, one at a time. Science 343, 1083-1084 (2014).

37. Kim, H. et al. Identification of single-atom $\mathrm{Ni}$ site active toward electrochemical $\mathrm{CO}_{2}$ conversion to CO. J. Am. Chem. Soc. 143, 925-933 (2021).

38. Lin, L. et al. Enhancing $\mathrm{CO}_{2}$ electroreduction to methane with a cobalt phthalocyanine and zinc-nitrogen-carbon tandem catalyst. Angew. Chem. Int, Ed. 59, 22408-22413 (2020).

39. Collman, J. P., Brauman, J. I., Halbert, T. R. \& Suslick, K. S. Nature of $\mathrm{O}_{2}$ and $\mathrm{CO}$ binding to metalloporphyrins and heme proteins. Proc. Natl Acad. Sci. U. S. A 73, 3333-3337 (1976).

40. Caruso, M. M. et al. Mechanically-induced chemical changes in polymeric materials. Chem. Rev. 109, 5755-5798 (2009).

41. Boucly, A. et al. Soft X-ray heterogeneous radiolysis of pyridine in the presence of hydrated strontium-hydroxyhectorite and its monitoring by nearambient pressure photoelectron spectroscopy. Sci. Rep. 8, 6164 (2018).

42. Liou, K.-H., Yang, C. \& Chelikowsky, J. R. Scalable implementation of polynomial filtering for density functional theory calculation in PARSEC. Comput. Phys. Commun. 254, 107330 (2020).

43. Perdew, J. P. \& Wang, Y. Accurate and simple analytic representation of the electron-gas correlation energy. Phys. Rev. B 45, 13244-13249 (1992).

44. Troullier, N. \& Martins, J. L. Efficient pseudopotentials for plane-wave calculations. Phys. Rev. B 43, 1993-2006 (1991).

45. Ceperley, D. M. \& Alder, B. J. Ground state of the electron gas by a stochastic method. Phys. Rev. Lett. 45, 566-569 (1980).

\section{Acknowledgements}

The authors gratefully acknowledge Yeju Zhou, Dan Gregory, Michele L. Sarazen, and Guangming Cheng for help with data processing and general discussion. This work was partially supported by ExxonMobil through its membership in the Princeton E-filliates Partnership of the Andlinger Center for Energy and the Environment. This research made use of the Imaging and Analysis Center operated by the Princeton Institute for the Science and Technology of Materials at Princeton University, which is supported in part by the Princeton Center for Complex Materials, a National Science Foundation Materials Research Science and Engineering Center (Grant No. DMR-2011750). D.F. and J.R.C. acknowledge support from the Welch Foundation under grant F-1837 and the U.S. Department of Energy under DOE/DE-FG02-06ER46286. The National Energy Research Scientific Computing (NERSC) and the Texas Advanced Computing Center (TACC) provided computational resources.

\section{Author contributions}

P.C. and N.Y. designed and carried out the experiments. D.F. and J.R.C. performed the DFT calculations. A.S. and E.A.C. provided theoretical insight. P.C., N.Y., Y.Z., D.F., drafted the manuscript with the input from A.S., J.R.C., E.A.C., C.B.A., D.C.D and S.P.R. N.Y. directed the project. All authors discussed the results and contributed to the interpretation and conclusions.

\section{Competing interests}

The authors declare no competing interests.

\section{Additional information}

Supplementary information The online version contains supplementary material available at https://doi.org/10.1038/s41467-021-25932-6.

Correspondence and requests for materials should be addressed to Nan Yao, Yunlong Zhang or James R. Chelikowsky.

Peer review information Nature Communications thanks Yongsheng Leng, Jan van Ruitenbeek and the other, anonymous, reviewer for their contribution to the peer review of this work. Peer reviewer reports are available.

Reprints and permission information is available at http://www.nature.com/reprints

Publisher's note Springer Nature remains neutral with regard to jurisdictional claims in published maps and institutional affiliations.

(c) (i) Open Access This article is licensed under a Creative Commons C. Attribution 4.0 International License, which permits use, sharing, adaptation, distribution and reproduction in any medium or format, as long as you give appropriate credit to the original author(s) and the source, provide a link to the Creative Commons license, and indicate if changes were made. The images or other third party material in this article are included in the article's Creative Commons license, unless indicated otherwise in a credit line to the material. If material is not included in the article's Creative Commons license and your intended use is not permitted by statutory regulation or exceeds the permitted use, you will need to obtain permission directly from the copyright holder. To view a copy of this license, visit http://creativecommons.org/ licenses/by/4.0/.

(C) The Author(s) 2021 